\title{
Modelización y aplicación de las nuevas teorías del crecimiento económico regional en México
}

\author{
Mendoza Cota, Eduardo y Alejandro Díaz-Bautista (2006),

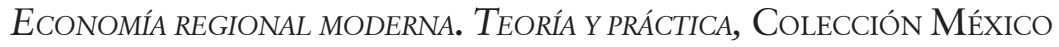 \\ Norte, Colegio de la Frontera Norte-Universidad de Guadala- \\ Jara-Plaza y Valdés, MÉxico, 190 PP., ISBN: 968-7947-46-2 \\ (Colef) ISBn: 970-722-483-5 (Plaza y Valdés)
}

La economía actual se genera con los nuevos conocimientos de las unidades territoriales y mediante una globalización integradora de los procesos económicos, sociales, culturales y territoriales, por tanto, un elemento característico hoy en día es la integración de los espacios económicos en el ámbito internacional.

Uno de los aspectos fundamentales a los que se enfrentan los economistas es estudiar la problemática económica desde una perspectiva regional en el proceso de globalización de la economía. Es decir, determinar cuáles son las tendencias de crecimiento y/o desarrollo que el mercado va fijando para las distintas regiones de un país y proponer políticas efectivas para revertir las tendencias negativas, así como analizar los efectos regionales de la inserción de los países a los acuerdos comerciales internacionales y al mundo en general.

México no es ajeno a esta problemática, la economía ha sufrido una serie de transformaciones que conllevan el surgimiento de un nuevo modelo de desarrollo basado en la descentralización de las actividades económicas. Transformaciones que son resultado de procesos como la apertura de la economía nacional al sistema económico mundial y la dificultad de las empresas mexicanas para adaptarse al conjunto de las naciones líderes en innovación y competitividad.

Ante esto, México se ha visto en la necesidad de plantear programas para regular y fomentar su desarrollo, para lo cual estableció como punto importante una serie de políticas territoriales en los diferentes ámbitos de gobierno para generar cambios en el enfoque y poner énfasis en la evolución del territorio.

Investigaciones como la que ahora reseñamos son de gran importancia tanto para los estudiosos de la economía regional como para los economistas en formación, no sólo por la exhaustiva revisión teórica que desarrollan Eduardo Mendoza y Alejan- 
dro Díaz-Bautista sobre las nuevas teorías del crecimiento regional, sino porque incorporan herramientas econométricas y estadísticas que les permiten generar modelos explicativos para analizar los fenómenos regionales en México, esto a pesar de las serias dificultades que se presentan para obtener datos de tipo desagregado confiables y homogéneos para estudiar el crecimiento económico regional.

El aporte fundamental de esta obra, desde mi punto de vista, es que retoma uno de los temas básicos en el ámbito económico mexicano y mundial: el estudio del crecimiento económico regional, el cual ha venido cobrando mayor importancia debido, sobre todo, a la interacción que se está dando entre los fenómenos globales y la importancia de la localización de las actividades económicas. Es importante resaltar que este trabajo vincula las teorías del crecimiento regional con los instrumentos de medición y aplicación para la estimación de los fenómenos regionales en nuestro país, lo que convierte a este libro en consulta obligada para todos aquellos interesados en la aplicación teórica-empírica de los problemas regionales en nuestro país.

Uno de los objetivos de esta obra, como bien señalan los autores, es "contribuir en la definición y divulgación de los principales enfoques teóricos modernos y de las nuevas técnicas cuantitativas y estadísticas para abordar los fenómenos económicos que se desarrollan en la actualidad, así como la presentación de aplicaciones empíricas para el caso de la economía mexicana, con el doble propósito de mostrar la utilidad de la teoría económica regional moderna y de describir los fenómenos económicos regionales recientes en México".

Para abordar el tema teoría y práctica del análisis económico regional, los autores presentan un texto que se compone de una introducción, dos apartados fundamentales, 22 cuadros estadísticos además de una extensa bibliografía.

En la "Introducción" se revisa la evolución de las teorías del crecimiento regional, haciendo énfasis en las hipótesis que sustentan, así como en sus principales supuestos, donde exponen de manera muy puntual y didáctica la teoría de la localización y la economía espacial, el modelo keynesiano de análisis regional, la teoría del desarrollo económico, el crecimiento económico, la convergencia entre regiones y la nueva geografía económica como las bases teóricas que dan sustento al análisis económico regional moderno.

El primer apartado tiene como objetivo analizar el crecimiento regional estático, para lo cual los autores estudian en un pri- 
mer momento la importancia-como herramienta de análisis regional- del modelo de la base exportadora al describir el funcionamiento regional en un contexto de apertura. Diseñan una metodología para aplicar este enfoque mediante la variable empleo regional para calcular coeficientes de localización; para ejemplificar dicha metodología utilizan el caso de Baja California. En un segundo momento presentan la matriz de insumo-producto como herramienta fundamental para las tareas de planificación, evaluación y ordenamiento regional y territorial, describiendo sus bondades como método para obtener información.

En el segundo apartado del texto, y el más relevante por sus aportaciones teóricas y metodológicas, Eduardo Mendoza y Alejandro Díaz-Bautista inician con el análisis del crecimiento económico regional a largo plazo o nuevos enfoques dinámicos, el cual dividen en cuatro subcapítulos: "Crecimiento económico regional y la hipótesis de la convergencia", "La generación de ideas como motor del crecimiento económico", "Externalidades regionales y crecimiento económico regional” y "Crecimiento y nueva geografía económica”.

Por lo que respecta al estudio del crecimiento económico regional y la hipótesis de la convergencia, retoman el modelo de Solow, donde el crecimiento económico lo determinan elementos exógenos que afectan la función de producción y el dinamismo de la acumulación de capital e incluyen una aplicación de la hipótesis de convergencia al condicional para el caso de la economía mexicana. Asimismo incluyen el fenómeno de la apertura comercial que experimentó la economía mexicana durante los años ochenta y noventa. Complementa este enfoque el análisis del modelo de crecimiento endógeno de Romer, donde se endogeniza el progreso tecnológico mediante la introducción de la búsqueda de nuevas ideas por parte de investigadores interesados en maximizar las ganancias. En este mismo apartado se presenta una aplicación de dicho modelo con la finalidad de analizar la producción de innovaciones tecnológicas para el caso de México en el periodo 1996-2000.

Por lo que respecta a las externalidades regionales, los autores mencionan que el concepto de externalidad se asocia al espacio geográfico y a la localización de actividades económicas. En la medida que los factores externos afecten los costos y beneficios de las empresas, las economías externas regionales, al igual que las internas, reducirán los costos de producción incrementando la productividad de las empresas. Así, los beneficios de estas externalidades derivan fundamentalmente de las implica- 
ciones de la localización. Asimismo, se presenta una serie de estimaciones empíricas sobre el efecto que tienen las externalidades regionales en el crecimiento

En la última parte del libro, Mendoza y Díaz-Bautista hacen un recuento de las diferentes teorías del desarrollo regional citando autores como Von Thûnen con su teoría de la localización, Weber con la localización industrial, Christaller con su teoría sobre sistema de ciudades, entre otros, donde destacan la reciente teoría de Paul Krugman, la cual se enfoca en los elementos y categorías de análisis novedosos en la explicación del surgimiento, desarrollo y declive de las estructuras regionales y urbanas. Al final de la obra se presenta un modelo de crecimiento urbano regional aplicado al caso de México.

Una conclusión muy importante a la que llegan los autores, y con la cual coincido ampliamente, es que el crecimiento económico regional se debe basar en la sustentabilidad social, integrando las expectativas y demandas del conjunto de la sociedad urbana regional mediante un análisis interdisciplinario e intersectorial que parta de un enfoque de crecimiento regional balanceado. En este contexto, el libro constituye un avance fundamental en el análisis de las nuevas teorías que explican los fenómenos económicos regionales.

Finalmente, tengo la seguridad de que este texto, como bien lo expresan los autores, sentará las bases para discutir y analizar los cambios que enfrentará la economía regional en México, tomando como punto de partida la aplicación de las nuevas teorías de la economía regional al estudio concreto de su realidad, mediante el uso de modelos econométricos y estadísticos, incidiendo en la construcción de las nuevas políticas necesarias en nuestro país para lograr el desarrollo económico de sus regiones. No puedo dejar de mencionar, como un aporte significativo, la extensa y bien seleccionada bibliografía que refleja el dominio que los autores tienen del tema.

Recibido: 6 de marzo de 2007. Aceptado: 3 de mayo de 2007.

Carmen Salgado-Vega Universidad Autónoma del Estado de México Correo-e: casa1961@yahoo.cm.mx 
Carmen Salgado Vega. Es doctora en economía por la Universidad de Salamanca, España. Profesora-investigadora de la Facultad de Economía de la Universidad Autónoma del Estado de México y miembro del Sistema Nacional de Investigadores, nivel I. Sus principales líneas de investigación son: desarrollo regional, mercado laboral y educación. Publicaciones más recientes: "Educación universitaria y mercado de trabajo", Contexto Económico, 11, UAEM, México, pp. 11-23 (2005); "Empleo y transición profesional en México", Papeles de Población, 44, UAEM, México, pp. 243-274 (2005); "Conceptualización teórica sobre el mercado de trabajo", Historia, Políticas y Procesos Educativos, 3, UAEM, México, pp. 3445 (2006). 